\title{
NEUROART
}

\section{Neuroarte e cefaleia: os enigmas nos afrescos de Michelangelo}

\section{Neuroart and headache: the enigmas in the Michelangelo's frescos}

\author{
Marcelo Moraes Valença, Luciana P. A. Andrade-Valença \\ Unidade Funcional de Neurologia e Neurocirurgia, Departamento de Neuropsiquiatria, Universidade Federal \\ de Pernambuco, Cidade Universitária, Recife, PE, Brazil
}

Valença MM, Andrade-Valença LPA. Neuroarte e cefaleia: os enigmas nos afrescos de Michelangelo.

Headache Medicine. $2011 ; 2(4): 212-5$

\begin{abstract}
RESUMO
Neuroarte é uma disciplina das Neurociências onde arte e ciências/medicina se misturam. Grandes nomes como Leonardo da Vinci, Michelangelo, Vesalius usaram da arte da ilustração para documentar a anatomia humana. Neste artigo comentamos sobre estruturas anatômicas ocultas nos afrescos de Michelangelo encontrados na Capela Sistina. Também mostramos imagens de dois homens com expressão de dor unilateral e agitação, sugerindo cefaleia em salvas.
\end{abstract}

Palavras-chave: Michelangelo; Cefaleia; Arte; Neuroarte; Capela Sistina; Cefaleia em salvas

\section{ABSTRACT}

Neuroart is a discipline of Neurosciences where there is an interrelationship between art and sciences. Great names such as Leonardo da Vinci, Michelangelo, Vesalius used the art of illustration to document the human anatomy. In the present article we are commenting about hidden anatomical structures found in Michelangelo's frescos of the Sistine Chapel. We also showed the imagem of two men, expressing unilateral pain and agitation.

Keywords: Michelangelo; Headache; Art; Neuroart; Sistine Chapel; Cluster headache

\section{INTRODUÇÃO}

Um dos grandes incentivadores da Neuroarte no Brasil é o nosso amigo neurocientista Norberto Garcia Cairasco, (1) do Departamento de Fisiologia da Faculdade de Medicina de Ribeirão Preto-USP. Nas capas dos exemplares das teses apresentadas por seus alunos são mostradas criações artísticas que valorizam o conteúdo de novos conhecimentos escritos e divulgados pelo doutorando ou mestrando no momento da defesa.

\section{GÊNIOS DAS ARTES VISUAIS DA RENASCENÇA}

Gênios das artes visuais da Renascença como Michelangelo (Michelagnolo) Buonarroti (1475-1564), Raphael Sanzio (1483-1520) e Leonardo da Vinci (14521519), bem como da Medicina [e.g. Vesalius (15141564) e Albinus (1697-1770)], deixaram registrados belos exemplos da Neuroarte.

Michelangelo pintou o afresco A Criação de Adão (280 cm x $570 \mathrm{~cm}$, Figura 1), por volta de 1511 , exposto no teto da Capela Sistina no Vaticano, que representa a criação de Adão por Deus. Estudiosos ${ }^{(2)}$ acreditam que a imagem pintada apresenta similaridades anatômicas com o encéfalo, podendo ser visualizados na posição lateral o lobo frontal, nervo ótico, glândula pituitária, tronco cerebral e o cerebelo.

Curiosamente o manto de Deus tem a forma de útero, e a charpe verde representaria o cordão umbilical 


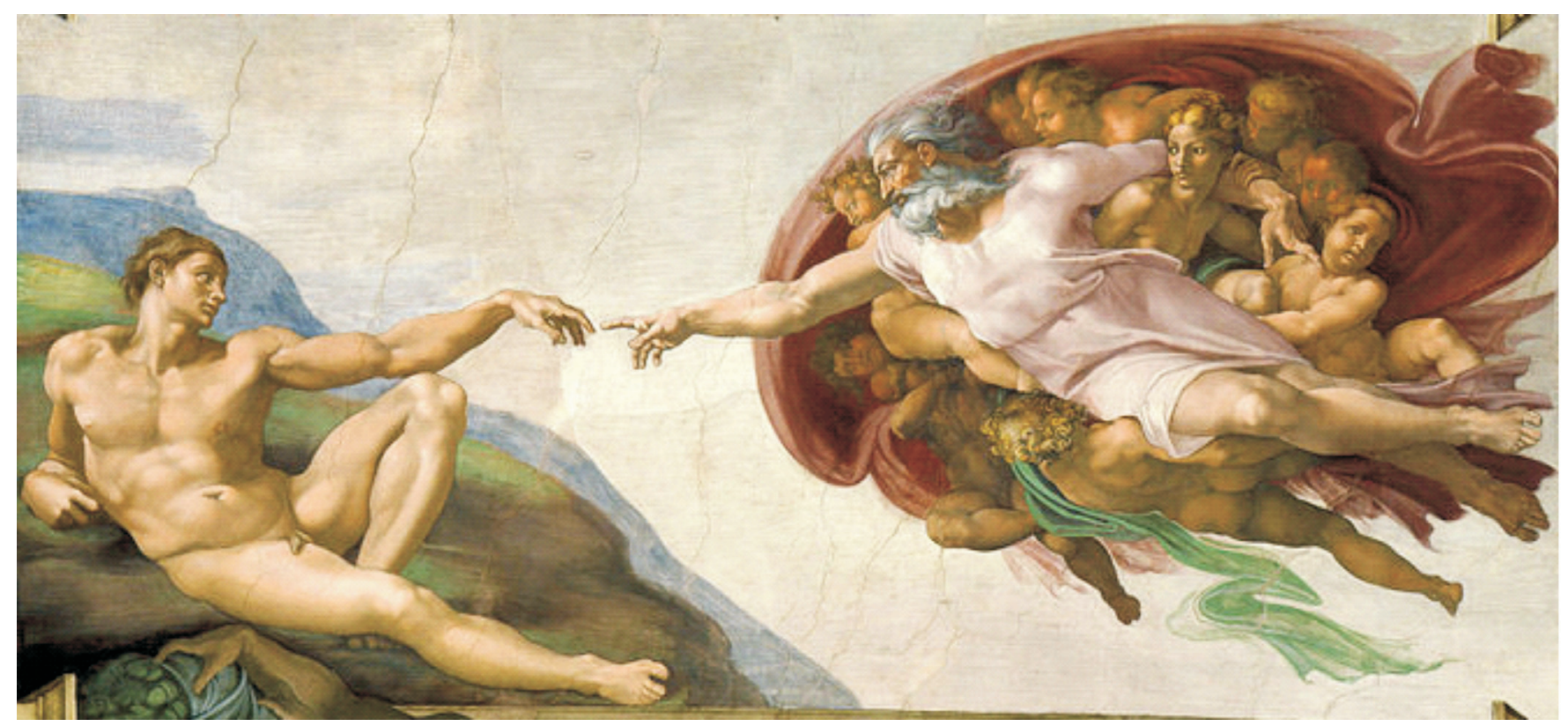

Figura 1. Afresco "A Criação de Adão" (1511), por Michelangelo. Ver o desenho do III ventrículo no abdome e do IV ventrículo do lado esquerdo do joelho direito. Seria esta imagem atrás de Adão a representação de uma mama, símbolo de nascimento e criação?

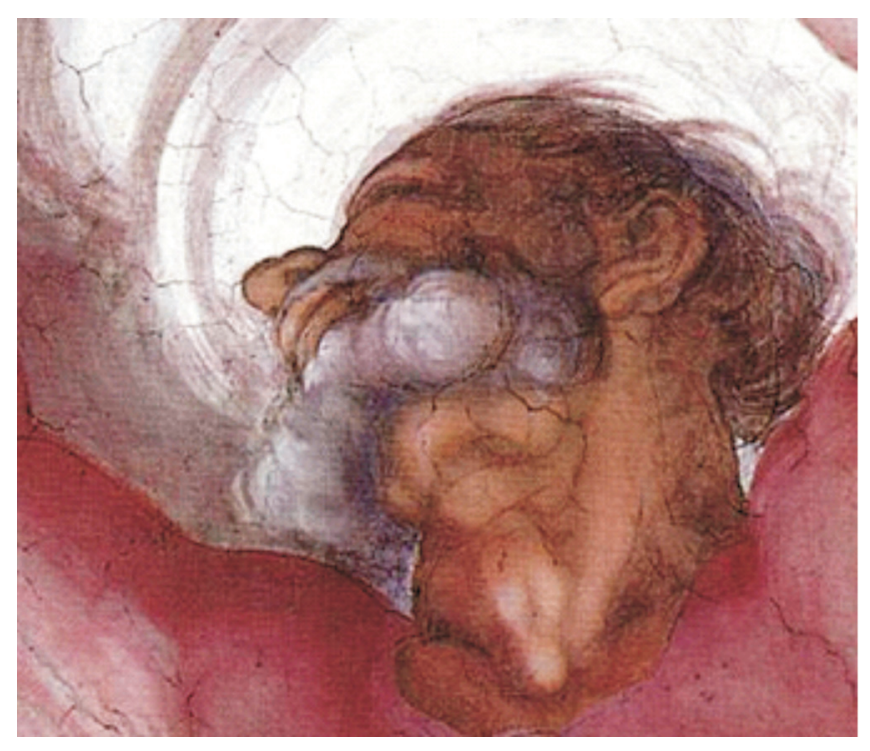

Figura 2. Cabeça de Deus no afresco "A Separação entre a Luz e as Trevas", por Michelangelo. Ver o desenho do tronco cerebral no pescoço de Deus

ou, segundo outros, as artérias vertebral e basilar. Todavia a nossa interpretação é diferente: o manto de Deus representa a aracnoide, membrana semitransparente que envolve todo o neuroeixo, e a charpe representa $o$ sifão carotídeo que continua com as artérias cerebral média e cerebral anterior. Ainda vizualizamos os forames de Monro e de Magendie, a cisterna quadrigêmea, e, no corpo de Deus, o aqueduto de Sylvius, os III e IV ventrículos cerebrais. Tentem identificar a artéria comunicante anterior, as artérias vertebrais, glândula pituitária, o nervo/quiasma óptico... entre outras estruturas cerebrais (Figura 1).

Será que Michelangelo intuitivamente também tentou representar uma "sinapse" ao desenhar a mão direita de Deus em direção à mão esquerda de Adão? Observem que há um mínimo espaço entre os dois dedos no intúito de vincular a imagem com transferência de informação (Figura 1).

Ainda no teto da Capela Sistina podemos encontrar a representação do tronco cerebral no pescoço de Deus no afresco A Separação entre a Luz e as Trevas (Figura 2). ${ }^{(3)}$

\section{TENTOU MICHELANGELO PINTAR UMA PESSOA COM CEFALEIA EM SALVAS?}

No afresco $O$ Juízo Final (Figura 3A), entre as inúmeras imagens de pessoas pintadas por Michelangelo encontra-se uma mulher, identificada como Santa Mônica, com exoftalmia, ${ }^{(4)}$ sugerindo oftalmopatia associada com doença tireoidiana, doença descrita só em 1835 por Graves. Duas outras imagens parecem representar homens com cefaleia, ambas sugerindo agitação e dor unilateral (Figura 3B e 3C). Estaria Michelangelo representando pessoas com cefaleia em salvas? 

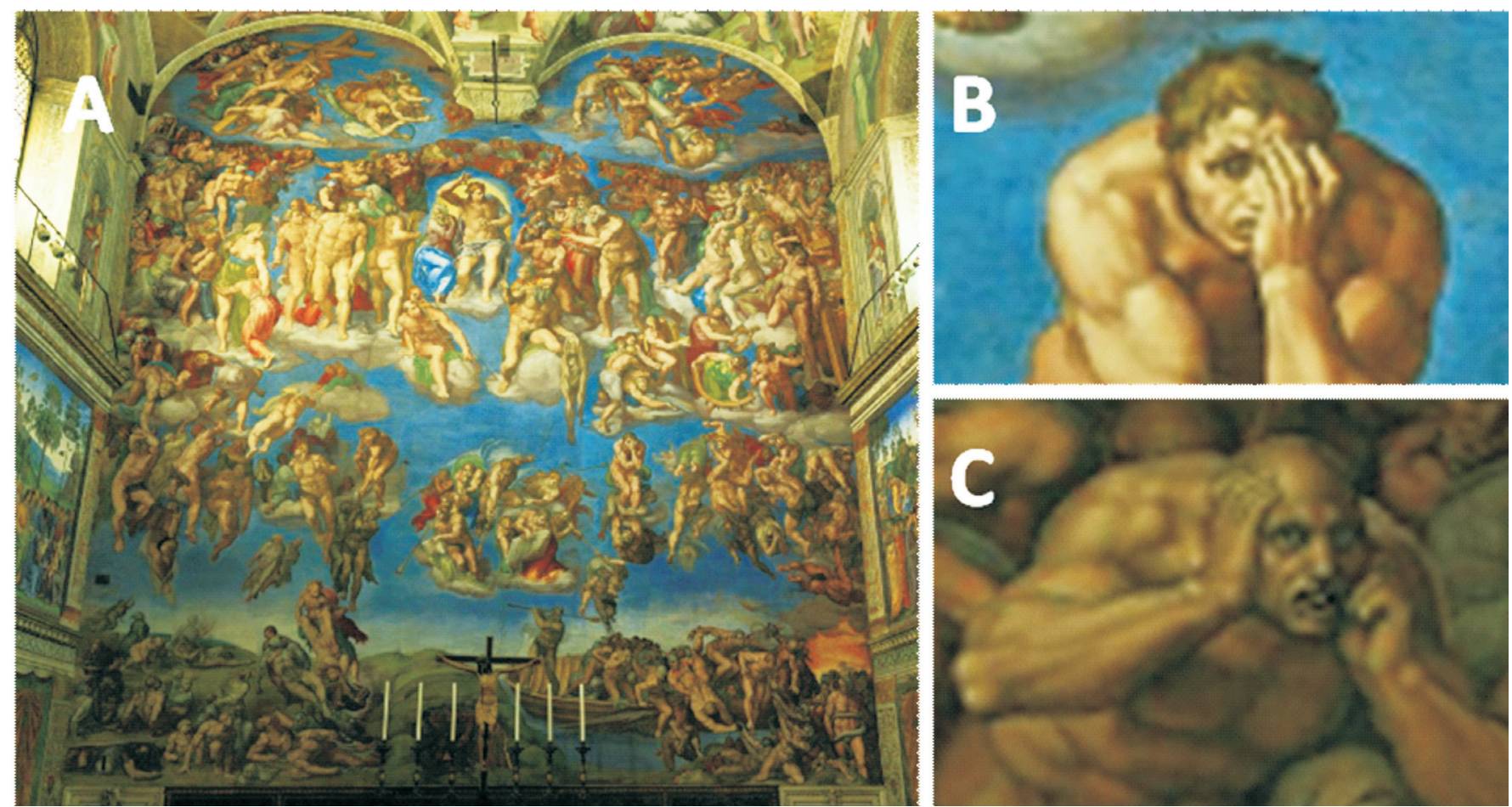

Figura 3. Afresco "A Separação entre a Luz e as Trevas", por Michelangelo (1537-1541)

\section{A CARICATURA DA DOR DE CABEÇA}

Neuroarte inclui trabalhos de artistas, que, de uma forma ou de outra, estejam vinculados com as neurociências, na pintura, literatura, música, teatro etc. Abaixo estamos mostrando a arte por pintura com o dedo sobre um prato executada pelo artista mineiro Vagner Bispo, quando foi solicitado na ocasião do XXV Congresso Brasileiro de Cefaleia, para pintar o que ele acreditava ser uma "dor de cabeça".

Sentado na rua 25 de março, na cidade de São Paulo, ele riu sem acreditar no que havia sido pedido, e cerca de 20 minutos depois podíamos ver a face de dor representada como uma "caricatura" de uma pessoa com cefaleia, onde a língua representava "o grito de dor". $\bigcirc$ artista Vagner não era um sofredor de cefaleia, mas sua tia, falou ele, "corria doida quando sua cabeça doía".

Um cefaliatra poderia identificar pistas de aura visual (escótomas cintilantes no quadrante superior esquerdo, turvação visual no quadrante superior direito e espectro de fortificação no brinco) e sinais autonômicos como hiperemia conjuntival, além de expressão de intensa dor, próprios de uma crise de enxaqueca.

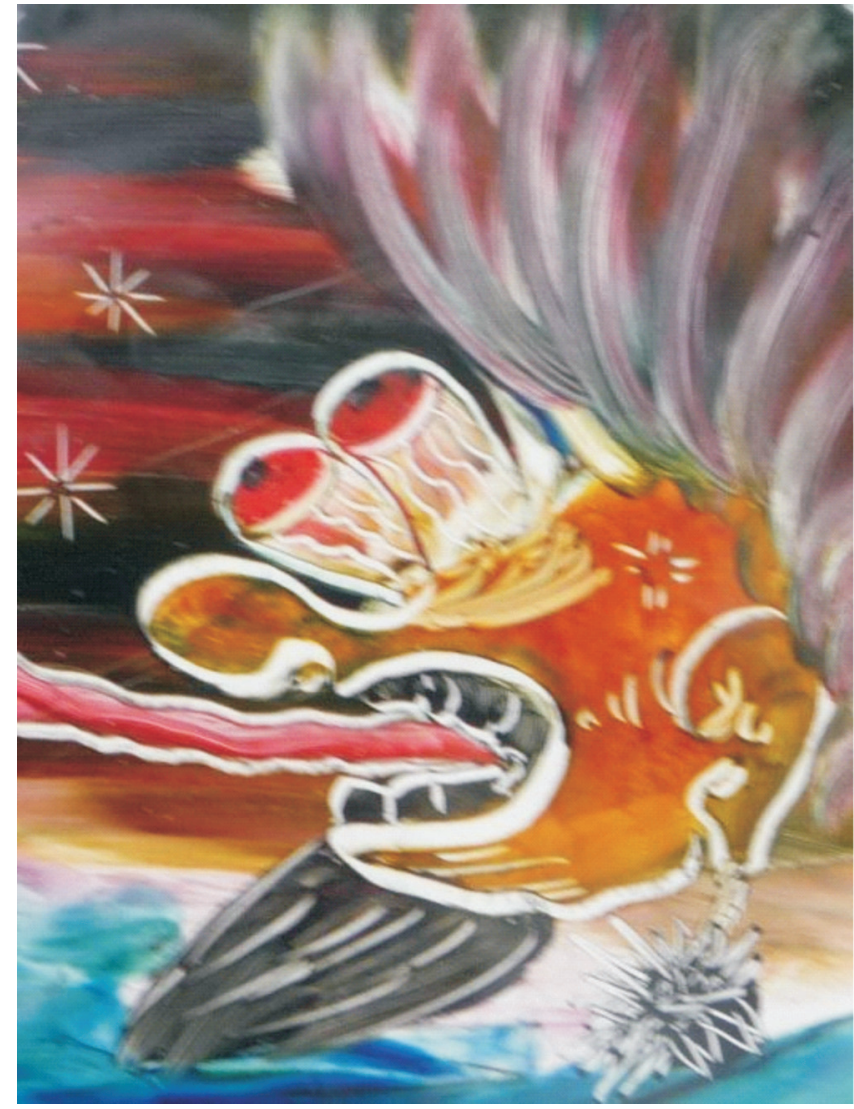

Figura 4. Dor de Cabeça, por Vagner Bispo (2011) 


\section{REFERÊNCIAS}

1. http://www.cerebromente.org.br/n10/opiniao/cairasco/art.html

2. Meshberger FL. An interpretation of Michelangelo's Creation of Adam based on neuroanatomy. JAMA. 1990; 264(14): 1837 41. Comment in: JAMA. $1991 ; 265(9): 1111$.

3. Suk I, Tamargo RJ. Concealed neuroanatomy in Michelangelo's Separation of Light From Darkness in the Sistine Chapel. Neurosurgery. 2010 May;66(5):851-61; discussion 860-1. Comment in: Neurosurgery. $2011 ; 68(6)$ :E1774-5. Neurosurgery. 2011 ;69(2):E503; author reply E503-5.

4. Pozzilli P. Blessed with exophthalmos in Michelangelo's Last Judgment. QJM. 2003;96(9):688-90.

Recebido: 11/28/2011

Aceito: 12/12/201 1

$\overline{\text { Correspondence }}$
Marcelo M. Valença, MD
Neurology and Neurosurgery Unit, Department of
Neuropsychiatry, Universidade Federal de Pernambuco
Cidade Universitária
$50670-420-$ Recife, PE, Brazil.
Phone: $+558199229394 ;+558134263501 ;$
Fax: +558121268539
mmvalenca@yahoo.com.br

